\section{Chemical Imaging with Infrared Microscopy}

William J. McCarthy

Thermo Electron Corporation, Molecular Spectroscopy, Madison, WI

bill.mccarthy@thermo.com

The ability of infrared microscopy to combine the chemical fingerprinting of FT-IR (Fourier Transform infrared) spectroscopy with the spatial resolving power of microscopy has led to its widespread use in numerous industries during the past two decades. The recent incorporation of imaging detectors into infrared microscopes has led to an increasing range of applications for the technique. The increase in spatial information provided by FT-IR imaging microscopes has also allowed image analysis techniques developed in light or electron microscopy to be utilized for the first time. This article will discuss FT-IR microspectroscopy and present some of the current applications.

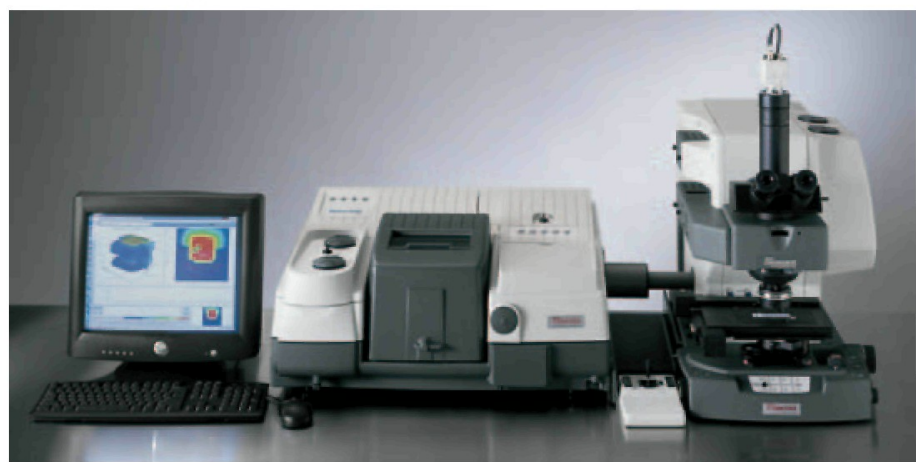

Figure 1. Nicolet Continuum XL Imaging Microscope with Nicolet 6700 FT-IR Spectrometer and OMNIC Atlus software

\section{Introduction}

FT-IR spectroscopic techniques have long been valued for the ability to uniquely identify the chemical signature of materials in a rapid, non-destructive analysis. There are innumerable applications in packaging material production, polymers, plastics, chemicals, raw materials, finished products, pharmaceuticals, forensics, paper (finished product and pulp), electronic materials, fillers, additives, coatings, fibers, biological cells and biological tissues.

Chemical imaging is increasing in importance as advances in materials are creating more sophisticated products. For example, a typical pharmaceutical tablet is no longer a compacted blend of a few ingredients. It is now a complex, spatially defined matrix of several active ingredients arranged in cores with fillers, lubricants, binders and barrier layers designed to dissolve in a predetermined sequence to deliver timereleased formulations with complex delivery profiles essential to proper function. Packaging materials are also becoming increasingly sophisticated with layers of polymers with different properties.

Chemical imaging has placed FT-IR into fields where the chemical specificity of FT-IR can be applied when the spatial distribution of the chemistry is essential for proper function. Important examples of these types of samples occur in biomedical ap plications that detect chemical changes in biological tissues. Recent studies have looked at cross-sections of skin, which has a complex spatial structure essential to proper function, and the chemical changes caused by applying cosmetics or drugs to the surface of the skin. Other studies have examined metastatic cancer in lymph nodes; chemical changes in bone tissue caused by arthritis, and degeneration of cartilage.

\section{Fourier Transform Infrared Spectroscopy}

The Michelson interferometer is the foundation of the modern FT-IR spectrometer. Due to the interferometer, FT-IR is very fast and sensitive. In less than a second, the interferometer creates an interferogram (interference pattern) that is transformed into a range of infrared frequencies corresponding to the chemical signature of the sample. Multiple scans can be averaged (known as coaddition) to improve signal-to-noise and still provide speedy analysis. An internal laser calibrates the infrared frequencies to provide highly accurate and reproducible spectra. These advantages allow sophisticated mathematical methods, such as database searching and chemometrics to be applied and provide the ability to examine small samples with mass in the picogram range and spatial dimensions down to the diffraction limit using an FT-IR microscope.

\section{FT-IR microscopes}

The first FT-IR microscopes (in the mid-1980s) allowed a single infrared spectrum to be obtained at any point on a sample using a single element detector. A single mask could be used to restrict the area measured. Later the ability to use two masks, then a single confocal aperture was developed that masked before and after the sample to provide ultimate spatial resolution by removing the effect of infrared diffraction caused by the sample.

These capabilities were maintained in mapping systems that incorporated an automated stage and software. This allowed the sequential collection of many points thus providing spatial distribution information of the chemistry of a sample. Mapping using a single element provides significant flexibility in sample analysis. Apertures can be adjusted to a larger area to improve signal, or small apertures can be overlapped to provide the ultimate achievable spatial resolu-

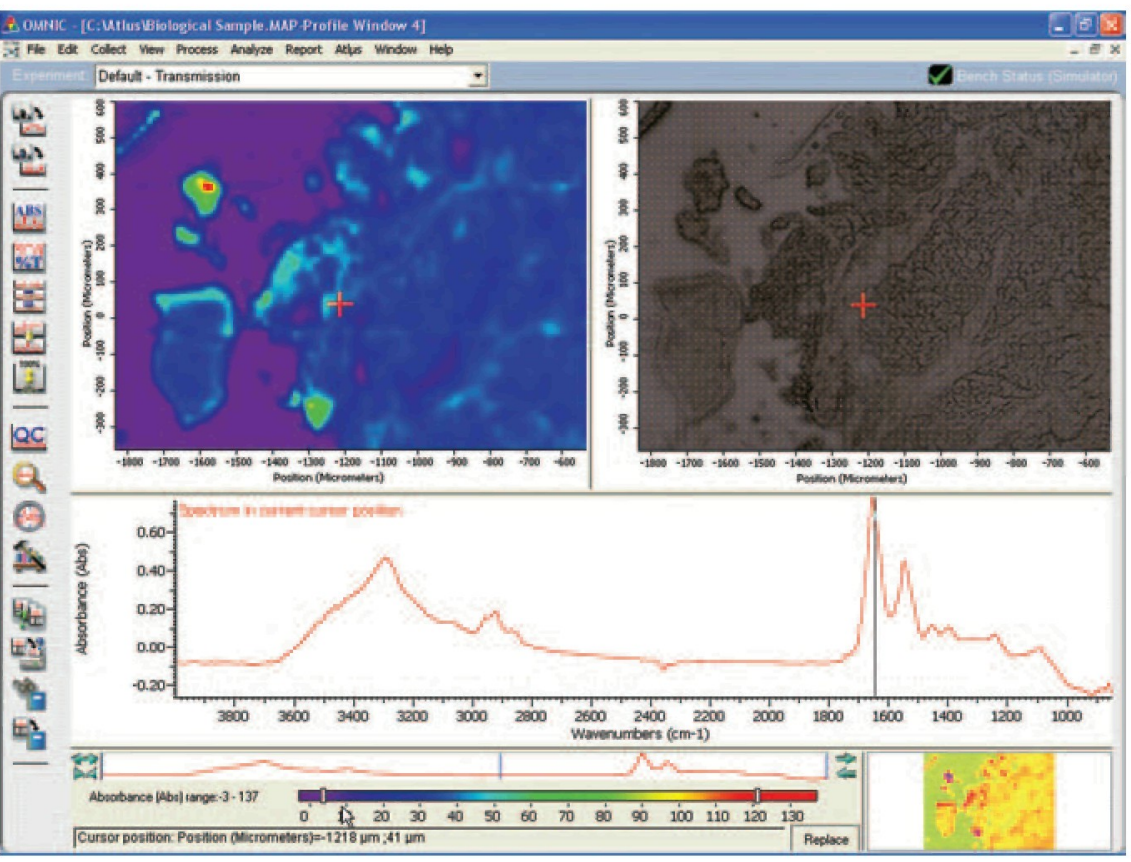

Figure 2. Managing, visualizing and analyzing chemical images requires a sophisticated and interactive software package. 


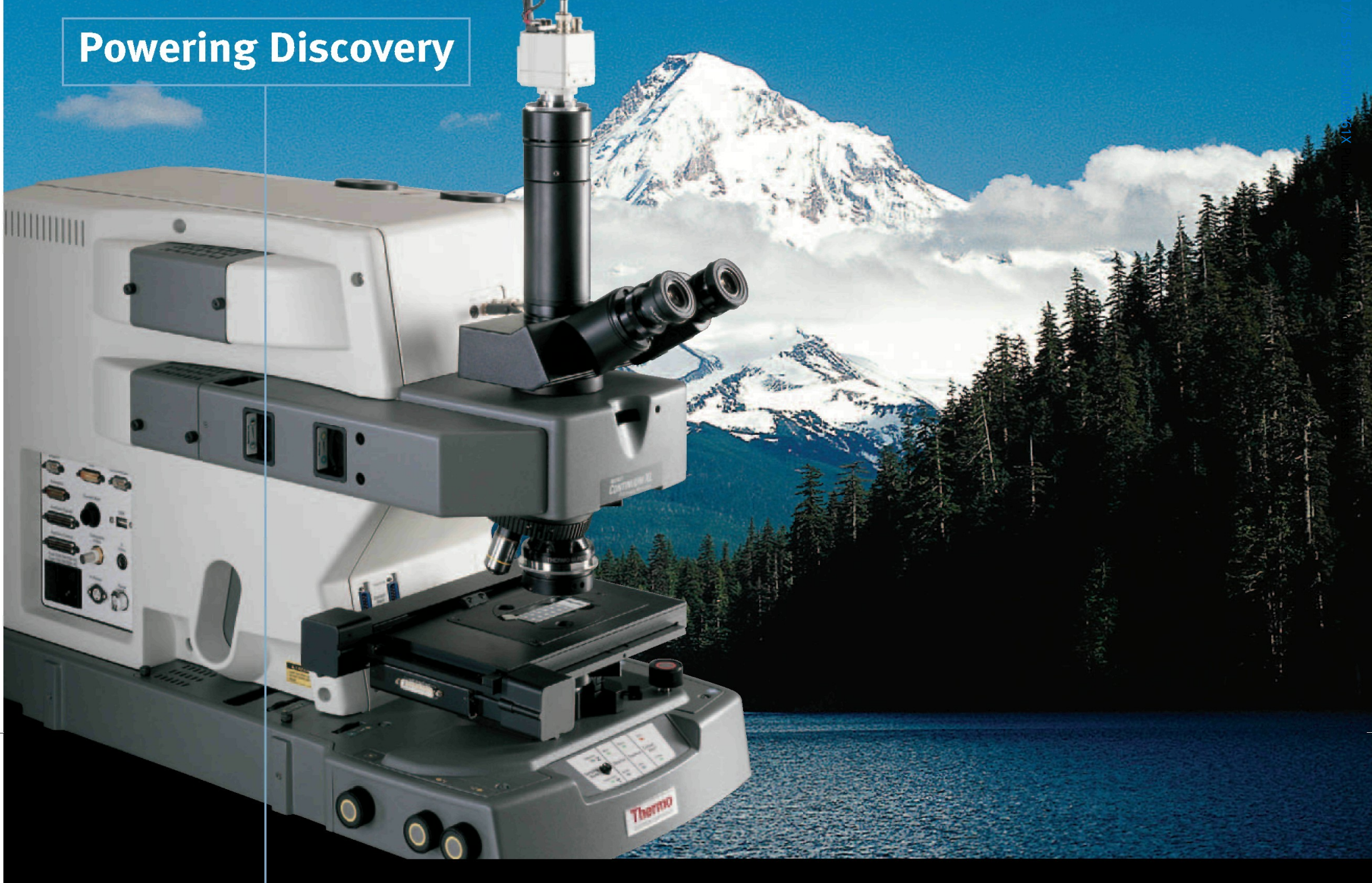

Nicolet Continu um XL FT-IR Imaging Microscope
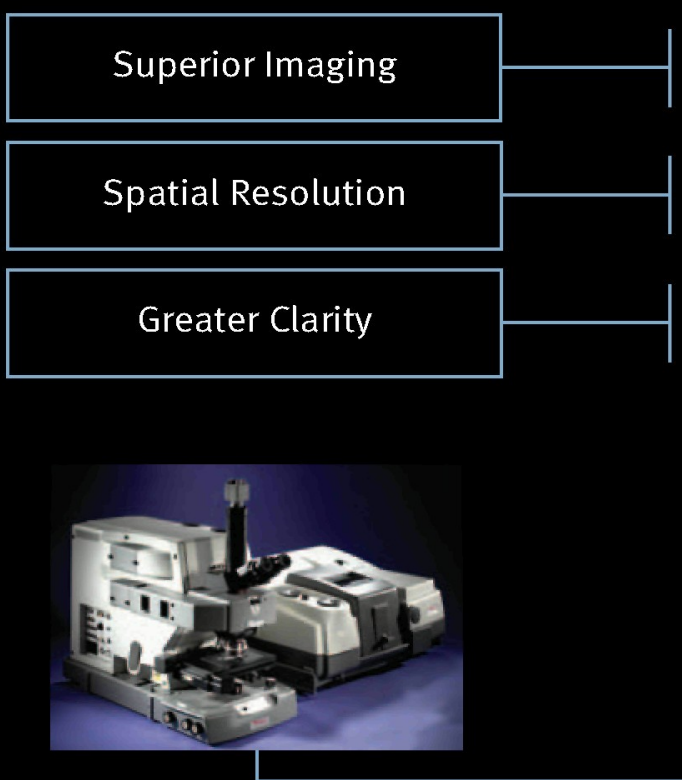

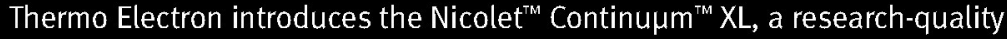
FT-IR microscope with the most advanced imaging available.

Unlock even your most mystifying sample. The innovative imaging design provides full spectral range with unprecedented image quality. Improve your throughput. A dual-masking remote aperture provides a continuous view of the sample while simultaneously collecting the purest spectral data.

Soar beyond current limitations. Couple the revolutionary imaging of this instrument with the power of Nicolet FT-IR spectrometers.

See the future of imaging today. Contact Thermo Electron at 1-800-532-4752, email analyze@thermo.com or visit www.thermo.com/ftir 


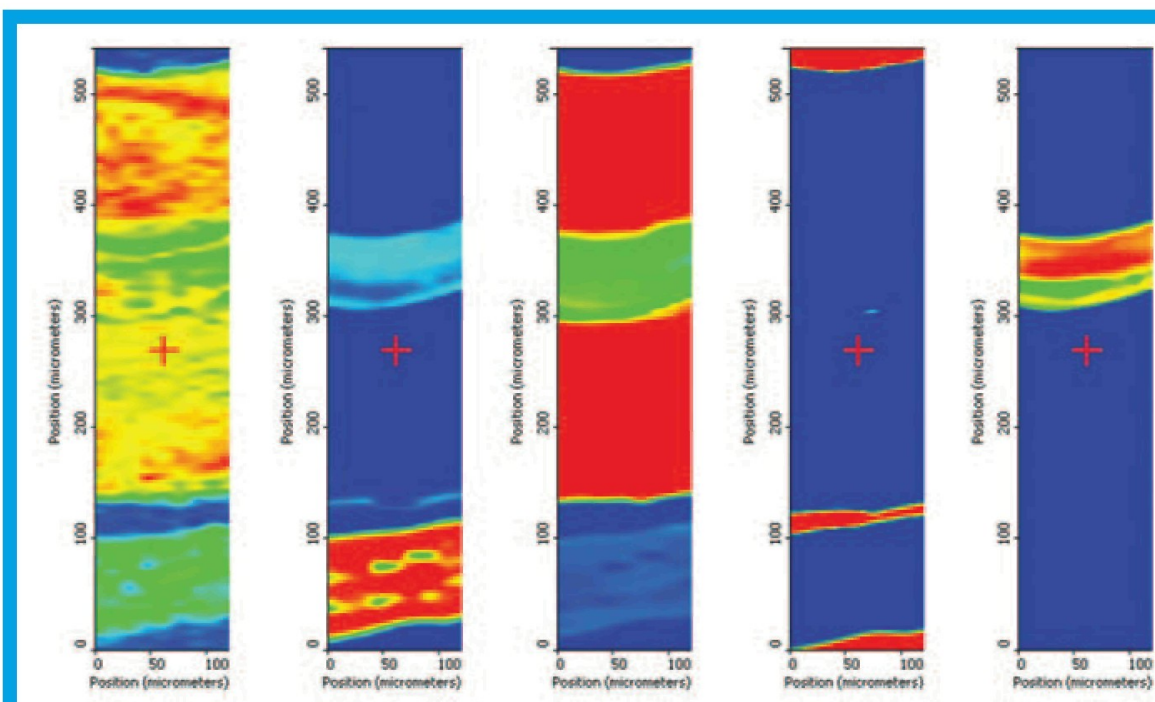

Multivariate chemometric techniques (for example principal component analysis or PCA), reveal the contribution and distribution of materials by reducing the thousands of individual wavelength images into several important images. Techniques such as correlation and discriminant analysis reduce the information to one important image. Multivariate curve resolution and PCA reconstruction use the chemical image information to produce spectra that can be more readily interpreted or searched.

Identifying the chemical composition of a sample is essential, but spatial distribution and dimensions also gives important information. For example, quantitative information could be obtained by identifying the areas of the active ingredient of a pharmaceutical tablet, summing the areas and determining the relative proportion. This determining and measuring of features has been a key component of image analysis

tion (albeit with a significant increase in analysis time). Also, if a large area of a sample needs to be covered, a single element detector does not have to cover every single point, but could just sample a few points to give a statistically valid assessment of spatial distribution. Single element detectors can be designed for different ranges and different sensitivities.

Imaging systems incorporated multi-element detectors that allow simultaneous collection of multiple spectra, building a chemical picture far faster than a mapping system. Most chemical imaging systems have the capability of using both an array detector and a single element detector and have the flexibility to perform single point, mapping and chemical imaging on one system. An example of this type of system is the Nicolet Continuum XL by Thermo Electron Corporation. It has a 28 element photoconductive linear array, with choice of infrared objectives with two magnifications, and the choice of five different types of single element detectors.

\section{Data Visualization and Analysis}

However the chemical picture is generated, each pixel in the image represents an entire spectrum of the sample area measured by the pixel. Since an average sized spectrum can have as many as a thousand frequency points, it is obvious that a chemical image consists of a huge amount of information. Managing, visualizing and analyzing the data requires a sophisticated and interactive software package.

The objective of FT-IR chemical imaging is to create specific chemical contrast that highlights compositional difference within a sample. Chemical imaging data can be viewed as multiple spectra arranged spatially or as a series of spatially resolved images produced at very narrow wavelength range. The analysis needs to be specifically designed to address the spectroscopic requirements and also portray the visual information. The simplest visualization software allows a user to view the data in both modes. The images are produced in false color in flat and three-dimensional perspective. More advanced techniques sum multiple wavelength regions (known as peak-picking) to improve signal-to-noise, and provide baseline correction and processing of derivatives to remove image artifacts and improve chemical contrast.

can be more readily interpreted or searched.
of visual microscopes for some time. The large sizes of chemical images obtained from novel FT-IR microscopes allows the algorithms of classical image analysis to be complimentary chemical analysis. Some of the algorithms applied allow direct measurement,

0.900 .951 .00

polyamide, $c$ )

\author{
(1)
} image feature extraction, filtering to enhance contrast, edge detection, surface definition, binarization of the image, feature sizing and

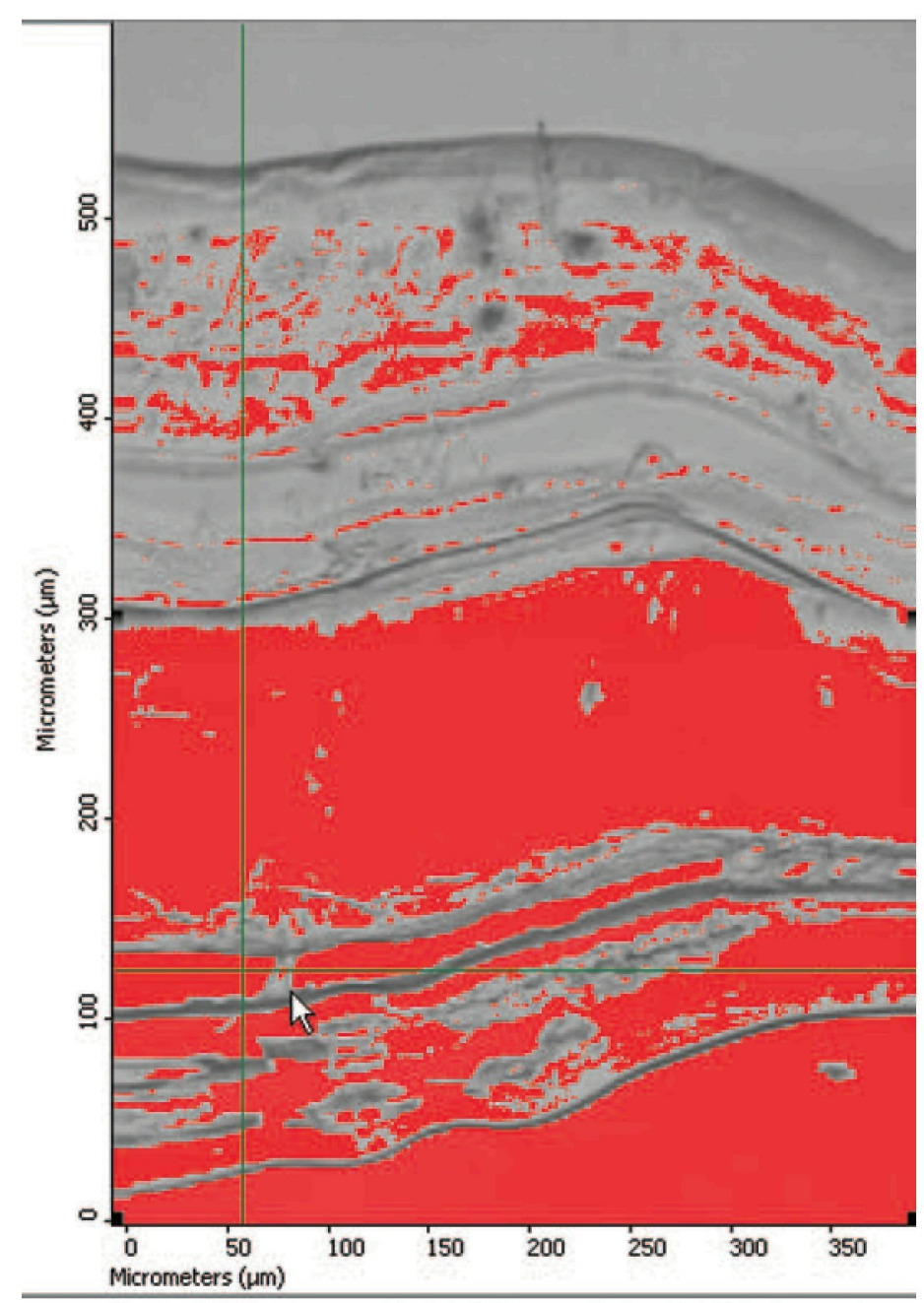

Figure 4. Video image with Sobel filter applied to highlight feature in the delaminated area 


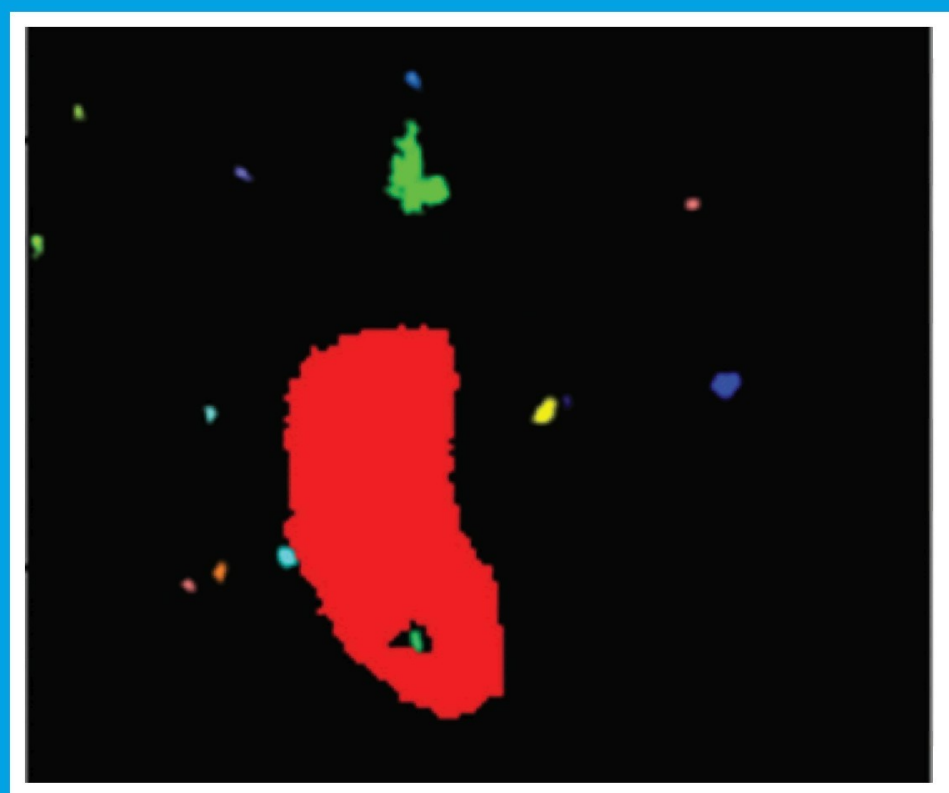
color

Figure 5. Binarized chemical image with particles identified by calculation of image statistics.

An example of such a software package can be seen in figure 2 . Thin sections of prepared human tissue were placed on a reflective low e-glass slide for infrared analysis. On the upper right corner is a video image of a sample. Below that is the spectrum associated with the pixel selected by the software. This spectrum indicates that this area of tissue has protein characteristics. A moveable bar in the spectrum window selects the frequency. To the left is a false color display of the absorbance intensities at a particular infrared frequency. The blue or cool colors show low absorbance values and the hot or red color are high absorbance values. The amount of a material present is linearly proportional to absorbance, so low absorbance indicates low levels of protein. A color bar below the spectrum shows the range of absorbance values for that particular frequency.

\section{Practical Example}

Packaging materials are commonly composed of multiple layers. They can exhibit multiple defects and delamination for multiple reasons. One type of defect is known as a fisheye. While fisheyes look similar, they can be caused by a variety of factors such as contaminants or air bubbles. Fisheyes can also be difficult to observe, because they are often colorless. Delamination occurs when the bonding between layers fails. An example of a laminated packaging material was sliced with a knife and compressed in a diamond anvil cell in order to induce stress. An area of about $120 \times 600$ micrometers was analyzed. Visual inspection showed 7 layers.

The raw intensity image at 1730 wavenumbers shows some differences (Figure 3a). Further analysis correlation of the image to library spectra gave the images seen in Figure 3b-c. Areas of red show high similarity and strong similarity (high correlation) while blue show significant differences to the reference spectrum. Correlation to air in figure $3 \mathrm{~d}$ shows that what appears to be a layer is actually an area of delamination. Figure $3 e$ uses PCA to highlight the barrier layer between two polyethylene layers.

Using image analysis on the video image reveals some interesting information about the delaminated area. Sobel filtering was applied to the video image to enhance edge detection. Figure 4 shows a "bridge" going across the clear delaminated area. Measurement tools determine that it was 25 micrometers long and less than 10 micrometers wide. A single element detector with dual aperturing was required to measure the area and determine that it was the bonding material that remained attached to the polyamide and polyethylene layers but failed everywhere else.

Several fisheyes were also examined and determined to be contaminants with different chemical signatures from the surrounding material. Using feature sizing, the particles area, perimeter and circularity were determined. In material sciences, such statistical analyses of particles, zones or features present in a sample are invaluable in correlating quantitatively back to physically measured properties.

\section{Conclusions}

Chemical imaging has proven to be a valuable tool for spectroscopists. The recent incorporation of classical imaging analysis that can be applied to visual image from a CCD camera as well as the extracted chemical image is invaluable taking imaging beyond 'illustrative pictures' of samples, allowing the extraction of information.

With these advances, chemical imaging is being introduced into application areas where the analyst is not necessarily a spectroscopist. One example is in medical applications where researchers may be unable to interpret a spectrum, but are very familiar and comfortable using images as a diagnostic tool.

\section{General References}

1. Fourier Transform Infrared Spectrometry by Peter R. Griffiths, James A.

De Haseth. Wiley-Interscience; 2nd Ed edition (March 14, 1986)

2. Multivariate Image Analysis by Paul Geladi, Hans Grahn. John Wiley \& Sons (January 24, 1997)

3. Fundamentals of Light Microscopy and Electronic Imaging by Douglas B. Murphy. Wiley-Liss; John Wiley \& Sons (2001) 